\title{
Linear Morphea-Induced Atrophy Treated with Hyaluronic Acid Filler Injections
}

\author{
Adrienne N. Choksi, MD, AND JefFrey S. Orringer, MD*
}

The authors have indicated no significant interest with commercial supporters.

$\mathrm{L}$ inear morphea in an en coup de sabre pattern is a variant of localized scleroderma that develops on the frontal or frontoparietal scalp. Approximately two-thirds of cases occur before the age of 18 . The etiology is unknown, but the disease is thought to be most likely autoimmune in nature. Between $50 \%$ and $75 \%$ of these patients have been found to have a positive serum antinuclear antibody (ANA), although ANA positivity has not been noted to correlate with disease activity. ${ }^{1,2}$ In en coup de sabre morphea, trauma has also been cited as a possible trigger. ${ }^{3}$ Although lesions may be limited to the skin, en coup de sabre morphea is notorious for extracutaneous involvement, and lesions may extend deep into underlying tissues and may be associated with neurologic, ophthalmologic, and oral abnormalities. $^{4}$

Linear morphea may be a self-limited disease, and some regression or softening of skin lesions often spontaneously occurs, although complete resolution in en coup de sabre morphea is unusual. Treatment of active, fibrotic disease often involves a variety of topical or oral medications, phototherapy, or both. Unfortunately, even once disease activity has subsided, patients are often left with some degree of noticeable skin atrophy. Lesions may leave disfiguring atrophic scars that can be psychosocially distressing to patients. Surgical options are often limited, but the use of soft tissue augmentation with injectable fillers has been explored. We report a case of facial linear morphea in an en coup de sabre pattern successfully treated initially using ultraviolet A (UVA1) phototherapy for active disease followed by hyaluronic acid filler injections for tissue contouring.

\section{Case Report}

A 20-year-old man presented to our clinic with an approximately 4-year history of a linear depression on the left side of his forehead, extending onto the scalp. The area was asymptomatic but cosmetically disfiguring, and the patient was interested in treatment options to improve the appearance of the affected skin. He had been seen in our department 3 years before, at which time he was diagnosed with linear morphea in an en coup de sabre pattern, and treatment with UVA1 was recommended for the active, fibrotic phase of his disease. He underwent approximately 22 treatments with UVA1 phototherapy. During the time of his UVA1 treatment series, the patient's disease activity halted, and significant softening of the affected skin was noted, but he was ultimately left with a residual atrophic linear plaque on the left side of his forehead (Figure 1).

Because the patient's linear morphea was no longer felt to be inflammatory or active, no additional treatment with UVA1 or systemic agents was pursued. For the cosmetic disfigurement that remained, we recommended injection of a non-animal-based cross-linked hyaluronic acid soft tissue filler (Perlane) to smooth the contours at

* Both the authors are affiliated with Department of Dermatology, University of Michigan Medical School, Ann Arbor, Michigan 


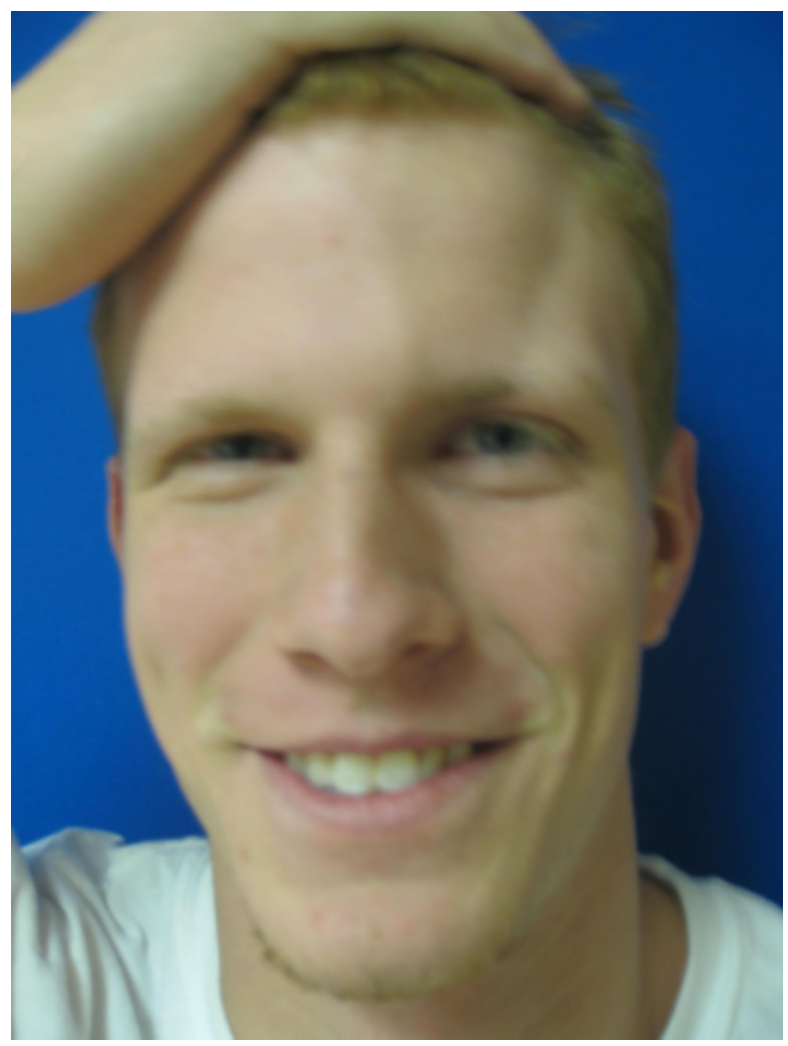

Figure 1. A 20-year-old man with atrophic scarring of the left side of his forehead secondary to linear morphea. The atrophy extends from the mid-brow into the hairline.

the involved site and thus improve the cosmetic appearance of the lesion. After local infiltration of small volumes of $1 \%$ lidocaine with epinephrine in a "ring block" pattern to achieve anesthesia, a total of $1 \mathrm{~mL}$ of hyaluronic acid was injected into the lesion using a linear threading technique, with an excellent aesthetic result, including approximately $75 \%$ improvement in the contour defect. (Only a single syringe was used during the initial treatment session at the patient's request.) Cosmetic improvements were immediate and sustained for at least 5 months. After 5 months, the patient continued to have stable improvement in the appearance of the atrophic skin, but a mild residual contour defect was still present (Figure 2). Therefore, a repeat injection of an additional $1 \mathrm{~mL}$ of hyaluronic acid was given to further enhance the cosmetic outcome (Figure 3). An improvement in the original contour defect esti- mated to be greater than $90 \%$ was achieved, and the patient was very pleased with this clinical result.

\section{Discussion}

Morphea en coup de sabre is a type of linear scleroderma that has a tendency to leave disfiguring atrophic scars. A variety of treatments have been used for linear morphea, with varying degrees of success. Treatments consist of those intended to inhibit the progression and inflammatory phase of the disease and those to treat the residual atrophy and disfigurement. Treatments used for the inflammatory phase of the disease include topical, intralesional, and systemic steroids; methotrexate; tacrolimus ointment; calcipotriol ointment; and phototherapy. UVA-1 has been found to be particularly effective in shortening the active period of the disease and preventing disease progression. ${ }^{5}$

There has been a relative paucity of therapeutic options for treating the residual atrophy that so often remains after the inflammatory phase of the disease has subsided. Previously described treatment modalities employed to improve the appearance of residual scarring include surgical procedures such as resection of the lesion, implantation of porous polyethylene implants, and AlloDerm tissue matrix. ${ }^{6-8}$ Although cosmetic improvements have been noted with such procedures, many of these treatment methods carry significant risks, require a substantial recovery period, or both. Other less-invasive treatment modalities have been also documented, with autologous fat grafting, tissue cocktail injections, and poly-L-lactic acid injections having been described in the literature. ${ }^{9-12}$ Autologous fat transfer requires harvesting of fat from a distant site and therefore necessitates an additional procedure, whereas the use of poly-L-lactic acid injections often requires several treatment sessions for optimal results and involves delayed cosmetic benefits. To our knowledge, cosmetic correction of scarring resulting from linear morphea in an en coup de sabre pattern using hyaluronic acid filler injections has not been previously reported. 

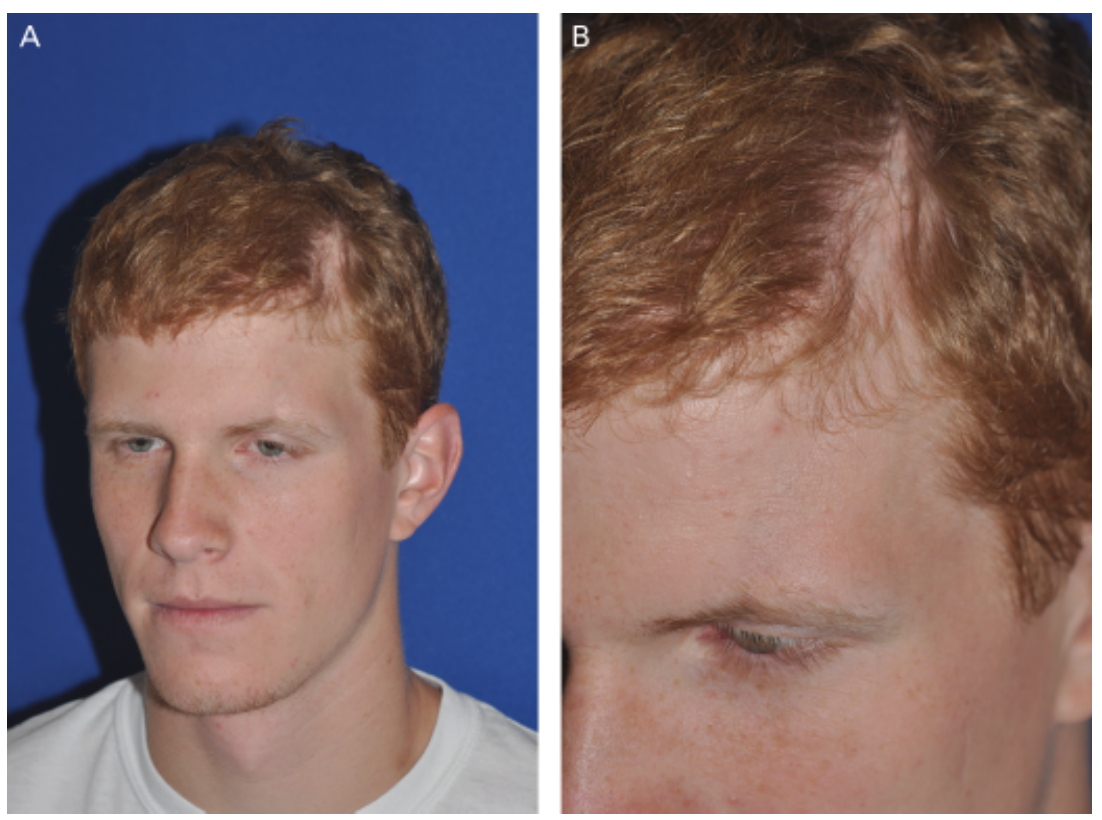

Figure 2. (A) Sustained aesthetic improvement 5 months after injection of hyaluronic acid. (B) Close-up view of treated area.

Hyaluronic acid fillers are typically used for correction of moderate to severe facial wrinkles and folds, for which they are FDA approved, although fillers are increasingly being used for off-label indications, including facial contouring. Initial trials involving another hyaluronic acid-based filler (Restylane) have suggested a 6-month duration of effect, and a more recent study showed persistent results up to 18 months after initial treatment with Restylane followed by one re-treatment session. ${ }^{13}$ In addition to

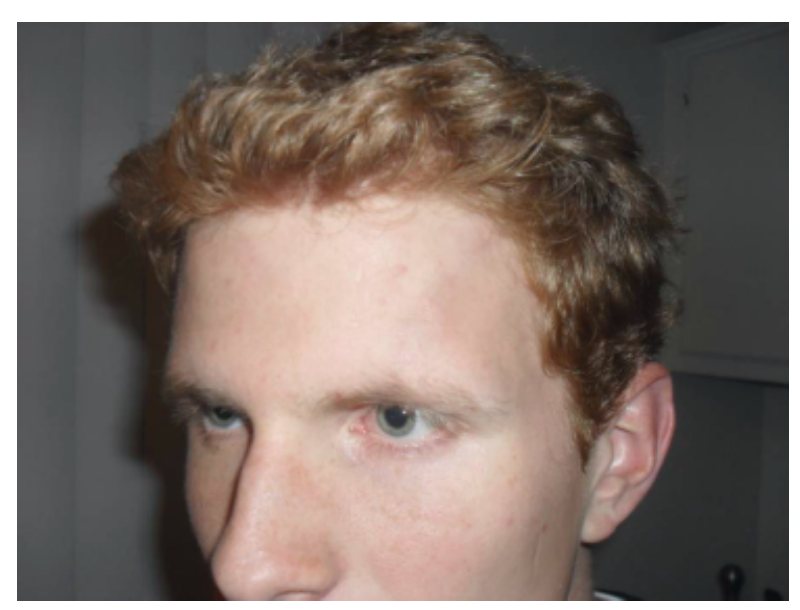

Figure 3. Enhanced aesthetic outcome 2 weeks after a second hyaluronic acid injection session. their immediate contouring effects, studies of hyaluronic acid fillers have demonstrated induction of type I collagen in the dermis, which may lead to prolonged cosmetic benefits. ${ }^{14}$ Although this may ultimately also prove to be true of other fillers, detailed molecular studies regarding this point are currently lacking. Perlane, with its relatively large particle size, is a robust hyaluronic acid filler shown to be highly useful in the treatment of a variety of soft tissue defects. ${ }^{15}$ It was selected in this case in the hope of achieving maximal aesthetic benefits with the smallest volume possible in our patient, who had financial constraints that limited the amount of filler we could use in a single treatment session. In addition, the theoretical reversibility of hyaluronic acid fillers due to the availability of hyaluronidase made Perlane an attractive treatment option.

Among the potential but rather uncommon complications of hyaluronic acid filler use are injection into or compression of vessels, causing tissue necrosis; persistent nodule or granuloma formation; allergic reactions; infection; and persistence of a visible blue hue. ${ }^{15}$ With respect to the first, care was taken to avoid inadvertent injection into the temporal or supraorbital vessels during the treatment. 
Reactivation of morphea due to antigenic stimulation with subsequent progression of the lesion constitutes a theoretical additional possible complication of hyaluronic acid filler injections for lesions of linear morphea. The benefits of hyaluronic acid filler injections for correction of scarring due to linear morphea include ease of injection, ready availability of adequate filler material, and the fact that it can be administered in a single treatment session on an outpatient basis with essentially no social downtime.

The cosmetic outcome in our patient has thus far been excellent. Our patient had developed a significant amount of softening of his lesion during treatment with UVA1 phototherapy, which allowed for maximal aesthetic benefits with the hyaluronic acid filler injections. It is probably wise to attempt correction of these lesions only once disease activity appears to be quiescent, although patients should be warned that the inflammatory phase of their disease could reactivate at any time, negating the effects of cosmetic corrective treatments, requiring the use of other medical therapies, or both. We believe that hyaluronic acid injections are an excellent therapeutic option for aesthetic improvement of scarring in linear morphea and may be a useful alternative to more invasive surgical procedures.

\section{References}

1. Marzano AV, Menni S, Parodi A, Borghi A, et al. Localized scleroderma in adults and children. Clinical and laboratory investigations in 239 cases. Eur J Dermatol 2003;13:171-6.

2. Arkachaisri T, Fertig N, Pino S, Medsger TA Jr. Serum autoantibodies and their clinical associations in patients with childhood- and adult-onset linear scleroderma. A single-center study. J Rheumatol 2008;35:2439-44.

3. Christen-Zaech S, Hakim MD, Afsar FS, Paller AS. Pediatric morphea (localized scleroderma): review of 136 patients. J Am Acad Dermatol 2008;59:385-96.
4. Holland KE, Steffes B, Nocton JJ, Schwabe MJ, et al. Linear scleroderma en coup de sabre with associated neurologic abnormalities. Pediatrics 2006;117:e132-6.

5. Kroft EBM, Berkhof NJG, van de Kerkhof PCM, Gerritsen RM, et al. Ultraviolet A phototherapy for sclerotic skin diseases: a systematic review.

J Am Acad Dermatol 2008;59:1017-30.

6. Dirschka T, Jackowski J, Bierhoff E, Folster-Holst R. Surgical correction of scleroderma en coup de sabre. Hautarzt 2007;58: 611-4.

7. Ozturk S, Acarturk TO, Yapici K, Sengezer M. Treatment of “en coup de saber" deformity with porous polyethylene implant. J Craniofac Surg 2006;17:696-701.

8. Robitschek J, Wang D, Hall D. Treatment of linear scleroderma "en coup de sabre" with AlloDerm tissue matrix. Otolaryngol Head Neck Surg 2008;138:540-1.

9. Lapiere JC, Aasi S, Cook B, Montalvo A. Successful correction of depressed scars of the forehead secondary to trauma and morphea en coup de sabre by en bloc autologous dermal fat graft. Dermatol Surg 2000;26:793-7.

10. Roh MR, Jung JY, Chung KY. Autologous fat transplantation for depressed linear scleroderma-induced facial atrophic scars. Dermatol Surg 2008;34:1659-65.

11. Oh C, Lee J, Jang B, Kang YS, et al. Treatment of atrophies secondary to trilinear scleroderma en coup de sabre by autologous tissue cocktail injection. Dermatol Surg 2003;29:1073-5.

12. Onesti MG, Troccola A, Scuderi N. Volumetric correction using poly-L-lactic acid in facial asymmetry: Parry Romberg syndrome and scleroderma. Dermatol Surg 2009;35:1368-75.

13. Narins RS, Dayan SH, Brandt FS, Baldwin EK. Persistence and improvement of nasolabial fold correction with non-animalstabilized hyaluronic acid 1,00,000 gel particles per $\mathrm{mL}$ filler on two retreatment schedules: results up to 18 months on two retreatment schedules. Dermatol Surg 2008;34Suppl 1:S2-8.

14. Wang F, Garza LA, Kang S, Varani J, et al. In vivo stimulation of de novo collagen production caused by cross-linked hyaluronic acid dermal filler injections in photodamaged human skin. Arch Dermatol 2007;143:155-63.

15. Beasley KL, Weiss MA, Weiss RA. Hyaluronic acid fillers: a comprehensive review. Facial Plast Surg 2009;25:86-94.

Address correspondence and reprint requests to: Jeffrey $S$. Orringer, MD, Department of Dermatology, University of Michigan Medical School, 1500 E. Medical Center Drive, 1910 Taubman Center, Ann Arbor, MI 48109, or e-mail: jorringe@umich.edu 